\title{
Compactness Properties of Weakly p-Harmonic Maps into Homogeneous Spaces
}

\section{Tatiana Toro \& Changyou Wang}

\begin{abstract}
In this paper we prove that the space of weakly $p$-harmonic maps from an open set in $\mathbf{R}^{n}$ into a homogeneous space with a left invariant metric is compact. We give a regularity criteria for weakly $p$-harmonic maps. As an application we prove that stationary $p$-harmonic maps into homogeneous spaces are regular, except possibly for a closed singular set of $(n-p)$-dimensional Hausdorff measure zero.
\end{abstract}

1. Introduction. In this paper we present some compactness results for weakly $p$-harmonic maps from an open subset of $\mathbf{R}^{n}$ into a compact homogeneous space, with a left invariant metric. In this context we prove that the the weak limit of a sequence of weakly $p$-harmonic maps is again a weakly $p$-harmonic map. We also show that stationary $p$-harmonic maps into homogeneous spaces are regular, except possibly for a closed singular set of $(n-p)$-dimensional Hausdorff measure zero. The proof crucially depends upon several observations of Hélein's and Shatah's, see [H1] and [Sh].

To state the results precisely, assume that $\Omega$ is a smooth open subset of $\mathbf{R}^{n}$, that $M$ is a smooth compact manifold and that $(N, h)$ is an $m$-dimensional smooth compact homogeneous space with a left invariant metric $h$. We also assume that $M$ and $N$ are isometrically embedded in some Euclidean space $\mathbf{R}^{k}$. A function $u$ in the Sobolev space $W^{1, p}\left(\Omega, \mathbf{R}^{k}\right)$ belongs to $W^{1, p}(\Omega, M)$ provided that $u(x) \in M$ a.e. in $\Omega$. Here $1<p \leq n$.

Definition. A function $u \in W^{1, p}(\Omega, M)$ is a weakly $p$-harmonic map of $\Omega$ into $M$ provided

$$
-\operatorname{div}\left(|D u|^{p-2} D u\right)=|D u|^{p-2} A(u)(D u, D u) \quad \text { weakly in } \Omega,
$$


where $A$ denotes the second fundamental form of the embedding $M \subset \mathbf{R}^{k}$. This system of partial differential equations is to hold in the weak sense, that is,

$$
\int_{\Omega}|D u|^{p-2} D u: D w d x=\int_{\Omega}|D u|^{p-2} A(u)(D u, D u) w d x
$$

for each test function $w \in W_{0}^{1, p}\left(\Omega, \mathbf{R}^{k}\right) \cap L^{\infty}\left(\Omega, \mathbf{R}^{k}\right)$. Here

$$
D u=\left(\frac{\partial u^{i}}{\partial x_{j}}\right)_{\substack{1 \leq j \leq n \\ 1 \leq i \leq k}}, \quad D u: D w=\frac{\partial u^{i}}{\partial x_{j}} \frac{\partial w^{i}}{\partial x_{j}} .
$$

Let $g: \partial \Omega \rightarrow M$ be a smooth function, then (1.1) is the Euler-Lagrange equation for the variational problem of minimizing the $p$-energy

$$
E_{p}[w]=\int_{\Omega}|D w|^{p} d x
$$

among functions $w \in W^{1, p}(\Omega, M)$ with $w=g$ on $\partial \Omega$ in the trace sense.

Under the previous assumptions we have:

Theorem 1. Let $(N, h)$ be a smooth compact homogeneous space, and $h$ a left invariant metric on $N$. Let $u_{k} \in W^{1, p}(\Omega, N)$ be a sequence of weakly $p$ harmonic maps, and assume that $u_{k} \rightarrow u$ in $W^{1, p}(\Omega, N)$. Then $u$ is a weakly p-harmonic map from $\Omega$ into $N$.

In general, very little can be said about the regularity of weakly $p$-harmonic maps. Helein [H2] proved that a weakly harmonic (i.e., $p=2$ ) map from $\Omega \subset \mathbf{R}^{2}$ into a smooth compact manifold is always smooth. On the other hand Rivière $[\mathrm{R}]$ constructed weakly harmonic maps from $B^{3}$ into the sphere $S^{2}$ which are singular in the whole $B^{3}$. Schoen and Uhlenbeck [SU] proved that that energy minimizing harmonic maps from $\Omega \subset \mathbf{R}^{n}$ into a smooth compact manifold are smooth, except possibly for a closed set of Hausdorff dimension at most $(n-3)$. Hardt and Lin $[\mathrm{HL}]$ proved a similar result for general $p$. Namely they showed that energy minimizing $p$-harmonic maps from $\Omega \subset \mathbf{R}^{n}$ into a smooth compact manifold are $C^{1, \alpha}$, for some $\alpha \in(0,1)$, except possibly for a closed set of Hausdorff dimension at most $(n-[p]-1)$.

If $u$ is a minimizer of $E_{p}[\cdot]$, then, in addition to (1.2), $u$ satisfies

$$
\left.\frac{d}{d t} E_{p}\left(u_{t}\right)\right|_{t=0}=0
$$

where $u_{t}(x)=u(x+t \zeta(x))$, and $\zeta \in C^{1}\left(\Omega, \mathbf{R}^{n}\right)$ any vector field with compact support in $\Omega$. 
Definition. A function $u \in W^{1, p}(\Omega, M)$ is a stationary $p$-harmonic map from $\Omega$ into $M$ if $u$ satisfies the identities (1.2), and (1.3) for all test functions $w$ and $\zeta$.

In particular, a stationary $p$-harmonic map satisfies the monotonicity inequalities

$$
\frac{1}{\sigma^{n-p}} \int_{B_{\sigma}(x)}|D u|^{p} d y \leq \frac{1}{\rho^{n-p}} \int_{B_{\rho}(x)}|D u|^{p} d y,
$$

for all concentric balls $B_{\sigma}(x) \subset B_{\rho}(x) \subset \Omega$ (see $\left.[\mathrm{P}]\right)$.

Evans [E1] (for $M=S^{m}$ ), and Bethuel [B1] (for $M$ a compact smooth manifold) proved that a stationary harmonic map $u$ from $\Omega$ into $M$ is smooth, except possibly for a closed set of $(n-2)$-dimensional Hausdorff measure zero. For stationary $p$-harmonic maps we prove the following theorem. We recently learned that this result was independently proved by Takeuchi [T1], in the case $M=S^{m}$.

Theorem 2. Let $(N, h)$ be a smooth compact homogeneous space, and $h$ a left invariant metric on $N$. Assume that $u \in W^{1, p}(\Omega, N)$ is a stationary p-harmonic map. Then there exists a closed subset $\mathcal{S} \subset \Omega$ such that

$$
\mathcal{H}^{n-p}(\mathcal{S})=0, \quad u \in C^{1, \alpha}(\Omega \backslash \mathcal{S}, N),
$$

for some $\alpha \in(0,1)$. Here $\mathcal{H}^{n-p}$ denotes the $(n-p)$-dimensional Hausdorff measure.

A natural question in this setting is whether the regularity is preserved when taking weak limits. Luckhaus [L1] proved that if $u_{k} \in W^{1, p}(\Omega, M)$ is a sequence of $p$-energy minimizing maps, where $M$ is a smooth compact manifold, and $u_{k} \rightarrow u$ weakly in $W^{1, p}(\Omega, M)$. Then $u$ is a $p$-energy minimizing map and hence the partial regularity results from either [SU] or [HL] apply.

Theorem 3. Let $(N, h)$ be a smooth compact homogeneous space, and $h$ a left invariant metric on $N$. Let $u_{k} \in W^{1, p}(\Omega, N)$ be a sequence of stationary p-harmonic maps, and assume that $u_{k} \rightarrow u$ in $W^{1, p}(\Omega, N)$. Then there exists a closed subset $\mathcal{S} \subset \Omega$ such that

$$
u \in C^{1, \alpha}(\Omega \backslash \mathcal{S}, N),
$$

for some $\alpha \in(0,1)$, and for any compact set $K \subset \Omega$

$$
\mathcal{H}^{n-p}(\mathcal{S} \cap K)<\infty \text {. }
$$

In the case $p=2$ Theorem 3 holds for any smooth compact target $M$. Moreover in this case standard elliptic regularity estimates (see $[\mathrm{M}]$ ) guarantee that the limiting map $u \in C^{\infty}(\Omega \backslash \mathcal{S}, N)$. 
Theorem 4. Let $M$ be a smooth compact manifold. Let $u_{k} \in W^{1,2}(\Omega, M)$ be a sequence of stationary harmonic maps, and assume that $u_{k} \rightarrow u$ in $W^{1,2}(\Omega, M)$. Then $u$ is a weakly harmonic map, and there exists a closed subset $\Sigma \subset \Omega$ such that

$$
u \in C^{\infty}(\Omega \backslash \Sigma, M),
$$

and for any compact set $K \subset \Omega$

$$
\mathcal{H}^{n-2}(\Sigma \cap K)<\infty
$$

Our proofs of Theorems 1,2 and 3 depend crucially upon the structure of the target manifold. The fact that $N$ is a homogeneous space with a left invariant metric guarantees that the right hand side of equation (1.1) belongs to the Hardy space $\mathcal{H}_{\text {loc }}^{1}(\Omega)$. We prove Theorem 1 in Section 2 . In Sections 3 and 4 we establish a regularity criteria for weakly $p$-harmonic maps into a homogeneous space with a left invariant metric. The main idea is to show that $u$ is continuous in a region where some analogue of the scaled energy is sufficiently small. In Section 5 we prove Theorems 2, 3 and 4 . Theorem 2 is a simple corollary of the Energy Decay Lemma proved in Section 3, it can also be deduced from Theorem 2.6 in [MY]. In order to prove Theorem 3 we show that if $u$ is the limit of stationary $p$-harmonic maps into a homogeneous space, then the regularity criteria described in Section 3 holds on a large region in $\Omega$. We would like to remark that the convergence statement from Theorem 4 was proved by Bethuel [B2], in the case $\Omega \subset \mathbf{R}^{2}$. For further results on weakly $p$-harmonic maps we refer the reader to [L2], [Fu1] and [Fu2].

2. Compactness Results. We start by recalling some relevant facts about homogeneous spaces with left invariant metrics, for more information see [CE]. Let $\left(N^{m}, h\right)$ be a smooth compact homogeneous space with a left invariant metric $h$. Let $X$ be a Killing tangent vector field on $N$, and let $u \in W^{1, p}(\Omega, N)$ be a weakly $p$-harmonic map. Then Noether's Theorem guarantees that the tangent vector field $|D u|^{p-2}\langle D u, X(u)\rangle$ defined on $\Omega$ is divergence free in the distribution sense. In fact since $u$ satisfies equation (1.1), for $\zeta \in C^{\infty}$ with compact support in $\Omega$, we have

$$
\int_{\Omega}\left\langle D_{\alpha}(\zeta X(u)),|D u|^{p-2} D_{\alpha} u\right\rangle d x=0 .
$$

We expand this equation to obtain

$$
\begin{aligned}
& \int_{\Omega} \zeta\left\langle D_{\alpha} X(u),|D u|^{p-2} D_{\alpha} u\right\rangle d x+\int_{\Omega} D_{\alpha} \zeta\left\langle X(u),|D u|^{p-2} D_{\alpha} u\right\rangle d x \\
= & \int_{\Omega} \zeta|D u|^{p-2}\left\langle D_{D_{\alpha} u} X(u), D_{\alpha} u\right\rangle d x+\int_{\Omega} D_{\alpha} \zeta\left\langle X(u),|D u|^{p-2} D_{\alpha} u\right\rangle d x=0 .
\end{aligned}
$$


But $\left\langle D_{D_{\alpha} u} X(u), D_{\alpha} u\right\rangle$ equals zero for any Killing vector field $X$. Therefore

$$
\int_{\Omega} D_{\alpha} \zeta\left\langle X(u),|D u|^{p-2} D_{\alpha} u\right\rangle d x=0,
$$

for any $\zeta \in C^{\infty}$ with compact support in $\Omega$, i.e.,

$$
\operatorname{div}\left(|D u|^{p-2}\langle X(u), D u\rangle\right)=0 \quad \text { weakly in } \Omega .
$$

Helein [H1] proved that under the previous assumptions there exist $q$ smooth tangent vector fields $Y_{1}, \ldots, Y_{q}$ and $q$ smooth Killing tangent vector fields $X_{1}, \ldots$, $X_{q}$ on $N$ such that for any $y \in N$ and for any tangent vector field $V$ in $T_{y} N$, we have:

$$
V=\sum_{i=1}^{q}\left\langle X_{i}, V\right\rangle Y_{i}
$$

A homogeneous space $N$ can always be identified with a quotient space $G / H$, where $G$ is a connected Lie group and $H$ is a closed subgroup. $q$ above represents the dimension of the Lie algebra of $G$. In particular, for each $\alpha=1, \ldots, n$,

$$
|D u|^{p-2} D_{\alpha} u=\sum_{i=1}^{q}|D u|^{p-2}\left\langle D_{\alpha} u, X_{i}\right\rangle Y_{i} .
$$

Computing the divergence on both sides and recalling that $|D u|^{p-2}\left\langle D u, X_{i}\right\rangle$ is a divergence free vector field, for each $i=1, \ldots, q$ we have

$$
\operatorname{div}\left(|D u|^{p-2} D u\right)=\sum_{\alpha=1}^{n} \sum_{i=1}^{q}|D u|^{p-2}\left\langle D_{\alpha} u, X_{i}(u)\right\rangle D_{\alpha} Y_{i}(u) \quad \text { weakly in } \Omega
$$

It is easy to check that a weak solution of (2.2) is also a weak solution of (1.1). Note that the right hand side of (2.2) is of the general form, the inner product of a gradient and a divergence free vector field.

We now recall some of the definitions and properties of the spaces BMO and $\mathcal{H}^{1}$, see [FS], [CLMS] and [S] for more information. If $f: \mathbf{R}^{n} \rightarrow \mathbf{R}$ is in $L_{\text {loc }}^{1}\left(\mathbf{R}^{n}\right)$, we set

$$
\|f\|_{*}=\sup \left\{\frac{1}{\left|B_{r}(x)\right|} \int_{B_{r}(x)}\left|f-(f)_{x, r}\right| d y: x \in \mathbf{R}^{n}, r>0\right\},
$$

where

$$
(f)_{x, r}=\frac{1}{\left|B_{r}(x)\right|} \int_{B_{r}(x)} f d y .
$$


We say that $f$ has bounded mean oscillation provided $\|f\|_{*}<\infty$, and refer to $\|f\|_{*}$ as the BMO norm of $f$. Assume now that $g \in L^{1}\left(\mathbf{R}^{n}\right)$. Define a class $\mathcal{T}$ of normalized test functions in $\mathbf{R}^{n}$ by

$$
\mathcal{T}=\left\{\varphi \in C^{\infty}\left(\mathbf{R}^{n}\right): \operatorname{supp} \varphi \subset B_{1}(0) \text { and }\|D \varphi\|_{L^{\infty}} \leq 1\right\} .
$$

We set

$$
g^{*}(x)=\sup _{t>0} \sup _{\varphi \in \mathcal{T}}\left|\varphi_{t} * g(x)\right|, \quad \text { where } \varphi_{t}(x)=t^{-n} \varphi\left(\frac{x}{t}\right),
$$

and we say that $g$ belongs to the Hardy space $\mathcal{H}^{1}\left(\mathbf{R}^{n}\right)$ if $g^{*} \in L^{1}\left(\mathbf{R}^{n}\right)$. We write

$$
\|g\|_{\mathcal{H}^{1}\left(\mathbf{R}^{n}\right)}=\left\|g^{*}\right\|_{L^{1}\left(\mathbf{R}^{n}\right)} .
$$

Note that $g \in \mathcal{H}^{1}\left(\mathbf{R}^{n}\right)$ implies $\int_{\mathbf{R}^{n}} g d x=0$.

A fundamental theorem of Fefferman $[\mathrm{F}]$ asserts that $\left(\mathcal{H}^{1}\right)^{*}=\mathrm{BMO}$ and establishes the inequality

$$
\left|\int_{\mathbf{R}^{n}} f g d x\right| \leq C\|f\|_{*}\|g\|_{\mathcal{H}^{1}\left(\mathbf{R}^{n}\right)}
$$

for $f \in L^{\infty}$ and $g \in \mathcal{H}^{1}\left(\mathbf{R}^{n}\right)$.

We now mention some results about $\mathcal{H}_{\text {loc }}^{1}(\Omega)$ which we use in the proof of Theorem 1, and in Section 4; for more information see [S]. Let $\Omega \subset \mathbf{R}^{n}$ be an open set, we say that $g \in L_{\text {loc }}^{1}(\Omega)$ lies in $\mathcal{H}_{\text {loc }}^{1}(\Omega)$ if for each compact set $K \subset \Omega$ there is an $\varepsilon>0$ so that

$$
\|g\|_{\mathcal{H}^{1}(K)}=\int_{K}\left(\sup _{0<t<\varepsilon} \sup _{\varphi \in \mathcal{T}}\left|\varphi_{t} * g(x)\right| d x\right)<\infty .
$$

Note that $\varphi_{t} * g(x)$ is defined for $\varphi \in \mathcal{T}$ as long as $t<\operatorname{dist}(x, \partial \Omega)$.

The following proposition relates $\mathcal{H}_{\text {loc }}^{1}(\Omega)$ to $\mathcal{H}^{1}\left(\mathbf{R}^{n}\right)$, allowing us to use the duality $\left(\mathcal{H}^{1}\right)^{*}=$ BMO "locally". For a proof see $[\mathrm{S}]$.

Proposition ([S]). Let $\Omega \subset \mathbf{R}^{n}$ be an open set, and let $g \in L_{\mathrm{loc}}^{1}(\Omega)$. Then $g \in \mathcal{H}_{\text {loc }}^{1}(\Omega)$ if and only if for each $C^{\infty}$ function $\vartheta$ with compact support contained in $\Omega$, and $\int \vartheta \neq 0$ there is a constant $\mu$ such that

$$
\vartheta(g-\mu) \in \mathcal{H}^{1}\left(\mathbf{R}^{n}\right) .
$$

Furthermore,

$$
\mu=\left(\int \vartheta\right)^{-1}\left(\int \vartheta g\right)
$$

and if $K=\operatorname{supp} \vartheta$,

$$
\|\vartheta(g-\mu)\|_{\mathcal{H}^{1}\left(\mathbf{R}^{n}\right)} \leq C_{K}\left(1+\|\vartheta(g-\mu)\|_{L^{1}\left(\mathbf{R}^{n}\right)}+\|g\|_{\mathcal{H}^{1}(K)}\right) .
$$

As remarked above, the right hand side of $(2.2)$ is of the general form the inner product of a gradient and a divergence free vector field. A result of Coifman, Lions, Meyer and Semmes [CLMS] guarantees then that this expression is in the Hardy space $\mathcal{H}_{\text {loc }}^{1}(\Omega)$. 
Proposition ([CLMS]). Let $\Omega \subset \mathbf{R}^{n}$. Assume $B \in W^{1, p}(\Omega), E \in$ $L^{p^{\prime}}\left(\Omega, \mathbf{R}^{n}\right)$, where $1 / p+1 / p^{\prime}=1$. Suppose also that $\operatorname{div} E=0$ in the distribution sense in $\Omega$. Then $\langle D B, E\rangle \in \mathcal{H}_{\mathrm{loc}}^{1}(\Omega)$, and for any $K \subset \Omega$ compact,

$$
\|\langle D B, E\rangle\|_{\mathcal{H}^{1}(K)} \leq C\left(\|D B\|_{L^{p}\left(\Omega, \mathbf{R}^{n}\right)}^{p}+\|E\|_{L^{p^{\prime}}\left(\Omega, \mathbf{R}^{n}\right)}^{p^{\prime}}\right) .
$$

Proof of Theorem 1. Let $\left\{u_{k}\right\}_{k \geq 1} \subset W^{1, p}(\Omega, N)$ be a sequence of weakly $p$-harmonic maps into a smooth compact homogeneous space with a left invariant metric. Assume that $u_{k} \rightarrow u$ weakly, $u \in W^{1, p}(\Omega, N)$. Since for $k=1,2, \ldots, u_{k}$ is a weak solution of

$$
\operatorname{div}\left(\left|D u_{k}\right|^{p-2} D u_{k}\right)=g_{k}
$$

where $g_{k}=\sum_{i=1}^{q}\left|D u_{k}\right|^{p-2}\left\langle D u_{k}, X_{i}\left(u_{k}\right)\right\rangle D Y_{i}\left(u_{k}\right)$, there exists a subsequence (which we reindex as necessary and denote also by $\left\{u_{k}\right\}_{k \geq 1}$ ) such that $u_{k} \rightarrow u$ strongly in $W^{1, p-1}(\Omega, N)$, so, in particular, $D u_{k} \rightarrow D u$ a.e. in $\Omega$ (see [HLM]), and $\left|D u_{k}\right|^{p-2} D u_{k} \rightarrow|D u|^{p-2} D u$ weakly in $L^{p^{\prime}}\left(\Omega, M^{k \times n}\right)$. Hence,

$$
g_{k} \rightarrow g=\sum_{i=1}^{q}|D u|^{p-2}\left\langle D u, X_{i}(u)\right\rangle D Y_{i}(u) \text { a.e. in } \Omega,
$$

with $g \in L^{1}(\Omega)$. Moreover, by the proposition above $g_{k} \in \mathcal{H}_{\text {loc }}^{1}(\Omega)$. Combining inequality (2.6) with the fact that $N$ is smooth and compact, we obtain the following estimate for any compact set $K$ in $\Omega$

$$
\begin{aligned}
&\left\|g_{k}\right\|_{\mathcal{H}^{1}(K)} \leq C \sum_{i=1}^{q}\left(\left\|D Y_{i}\left(u_{k}\right)\right\|_{L^{p}\left(\Omega, \mathbf{R}^{n}\right)}^{p}\right. \\
&\left.\quad+\left\|D u_{k}\right\|^{p-2}\left\|\left\langle D u_{k}, X_{i}\left(u_{k}\right)\right\rangle\right\|_{L^{p^{\prime}\left(\Omega, \mathbf{R}^{n}\right)}}^{p^{\prime}}\right) \\
& \leq C \int_{\Omega}\left|D u_{k}\right|^{p} d x .
\end{aligned}
$$

Let $\vartheta$ be a $C^{\infty}$ function with compact support in $\Omega$, and assume that $\int \vartheta \neq 0$. Then if $\mu_{k}=\left(\int \vartheta\right)^{-1}\left(\int \vartheta g_{k}\right) \in \mathbf{R}^{k}, \vartheta\left(g_{k}-\mu_{k}\right) \in \mathcal{H}^{1}\left(\mathbf{R}^{n}\right)$. Since

$$
\left|D u_{k}\right|^{p-2} D u_{k} \rightarrow|D u|^{p-2} D u \quad \text { weakly in } L^{p^{\prime}}\left(\Omega, M^{k \times n}\right),
$$

then using (2.6) and (2.9),

$$
\mu_{k}=\frac{\int \vartheta g_{k}}{\int \vartheta}=-\frac{\int\left|D u_{k}\right|^{p-2}\left\langle D u_{k}, D \vartheta\right\rangle}{\int \vartheta} \rightarrow-\frac{\int|D u|^{p-2}\langle D u, D \vartheta\rangle}{\int \vartheta},
$$




$$
G_{k}=\vartheta\left(g_{k}-\mu_{k}\right) \rightarrow G=\vartheta(g-\mu) \text { a.e. in } \mathbf{R}^{n},
$$

with

$$
\mu=-\frac{\int|D u|^{p-2}\langle D u, D \vartheta\rangle}{\int \vartheta},
$$

and $G=\vartheta(g-\mu) \in L^{1}\left(\mathbf{R}^{n}\right)$. Furthermore, if $K=\operatorname{supp} \vartheta$ for some $\varepsilon>0$, using (2.8)

$$
\begin{aligned}
\left\|G_{k}\right\|_{\mathcal{H}^{1}\left(\mathbf{R}^{n}\right)} & \leq C_{K}\left(1+\left\|\vartheta\left(g_{k}-\mu_{k}\right)\right\|_{L^{1}\left(\mathbf{R}^{n}\right)}+\left\|g_{k}\right\|_{\mathcal{H}^{1}(K)}\right) \\
& \leq C\left(1+\int_{\Omega}\left|D u_{k}\right|^{p} d x\right),
\end{aligned}
$$

where $C=C(\vartheta, N, n)$. Since $\left\{u_{k}\right\}_{k \geq 1}$ is uniformly bounded in $W^{1, p}(\Omega, N)$, then $\left\{G_{k}\right\}_{k \geq 1}$ is uniformly bounded in $\mathcal{H}^{1}\left(\mathbf{R}^{n}\right)$. We can apply the following theorem by Jones and Journé [JJ].

Theorem ([JJ]). Let $\left\{G_{k}\right\}_{k \geq 1}$ be bounded in $\mathcal{H}^{1}\left(\mathbf{R}^{n}\right)$. Assume that $\left\{G_{k}\right\}_{k \geq 1}$ converges a.e. to some $G \in L^{1}\left(\mathbf{R}^{n}\right)$. Then $G \in \mathcal{H}^{1}\left(\mathbf{R}^{n}\right)$ and $G_{k}$ converges to $G$ in the weak-* topology of $\mathcal{H}^{1}\left(\mathbf{R}^{n}\right)$.

In particular, for $G_{k}=\vartheta\left(g_{k}-\mu_{k}\right)$ as above, if $w \in L^{\infty}\left(\Omega, \mathbf{R}^{k}\right)$ with compact support in $\Omega$, then

$$
\int G_{k} w d x=\int \vartheta\left(g_{k}-\mu_{k}\right) w d x \rightarrow \int G w d x=\int \vartheta(g-\mu) w d x .
$$

We finally have all the necessary ingredients to finish the proof of Theorem 1 . Let $w \in W^{1, p}\left(\Omega, \mathbf{R}^{k}\right) \cap L^{\infty}\left(\Omega, \mathbf{R}^{k}\right)$ be a test function with compact support $K \subset \Omega$. Let $\vartheta$ be a smooth function with compact support in $\Omega$, assume $\vartheta=1$ on $K$, and $0 \leq \vartheta \leq 1$. Since each $u_{k}$ satisfies (2.2) we have, using the notation above,

$$
\begin{aligned}
-\int_{\Omega}\left|D u_{k}\right|^{p-2} D u_{k}: D w d x & =\int_{\Omega} g_{k} w d x \\
& =\int_{\Omega} \vartheta\left(g_{k}-\mu_{k}\right) w d x+\int_{\Omega} \vartheta \mu_{k} w d x \\
& =\int_{\Omega} G_{k} w d x+\int_{\Omega} \vartheta \mu_{k} w d x
\end{aligned}
$$


Letting $k$ go to infinity and applying (2.8), (2.10), (2.11) and (2.12), we conclude that

$$
\begin{aligned}
-\int_{\Omega}|D u|^{p-2} D u: D w d x & =\int_{\Omega} G w d x+\int_{\Omega} \vartheta \mu w d x \\
& =\int_{\Omega} \vartheta(g-\mu) w d x+\int_{\Omega} \vartheta \mu w d x \\
& =\int_{\Omega} g w d x \\
& =\int_{\Omega}\left(\sum_{i=1}^{q}|D u|^{p-2}\left\langle D u, X_{i}(u)\right\rangle D Y_{i}(u)\right) w d x .
\end{aligned}
$$

3. Energy Decay. Let $N$ be a smooth compact homogeneous space with a left invariant metric. Letting $u \in W^{1, p}(\Omega, N)$ for $B_{\rho}(x) \subset \Omega$, we define

$$
N(x, \rho, u)=\sup \left\{\frac{1}{r^{n-p}} \int_{B_{r}(y)}|D u|^{p}: B_{r}(y) \subset B_{\rho}(x)\right\}
$$

Note that for $0<\sigma \leq \rho, N(x, \sigma, u) \leq N(x, \rho, u)$, and so in particular

$$
N(x, u)=\lim _{\rho \rightarrow 0} N(x, \rho, u) \text { exists. }
$$

The key to the proof of Theorem 2 is the following decay lemma for the quantity defined above, which should be interpreted as the analogue of the scaled energy for stationary $p$-harmonic maps.

Decay Lemma 3.1. There exist constants $\varepsilon_{0}>0$ and $\tau \in\left(0, \frac{1}{16}\right)$ such that if $u \in W^{1, p}(\Omega, N)$ is a weakly $p$-harmonic map, then

$$
N(x, \rho, u) \leq \varepsilon_{0}
$$

implies

$$
N(x, \tau \rho, u) \leq \frac{1}{2} N(x, \rho, u)
$$

for $x \in \Omega, B_{\rho}(x) \subset \Omega$. 
Proof. We argue by contradiction, for $\tau>0$ selected as below. Were the lemma false there would exist balls $B_{\rho_{m}}\left(x_{m}\right) \subset \Omega$ such that

$$
N\left(x_{m}, \rho_{m}, u\right)=\lambda_{m}^{p} \rightarrow 0,
$$

whereas

$$
N\left(x_{m}, \tau \rho_{m}, u\right)>\frac{1}{2} \lambda_{m}^{p}
$$

Thus there exists $B_{\sigma_{m}}\left(y_{m}\right) \subset B_{\tau \rho_{m}}\left(x_{m}\right)$ such that

$$
\frac{1}{\sigma_{m}^{n-p}} \int_{B_{\sigma_{m}}\left(y_{m}\right)}|D u|^{p} \geq \frac{1}{4} \lambda_{m}^{p}
$$

Note that $B_{\sigma_{m} / 4 \tau}\left(y_{m}\right) \subset B_{\rho_{m} / 2}\left(x_{m}\right)$. For $r_{m}=\sigma_{m} / 4 \tau$ and $\eta=4 \tau \in\left(0, \frac{1}{4}\right)$, we have

$$
\frac{1}{r_{m}^{n-p}} \int_{B_{r_{m}}\left(y_{m}\right)}|D u|^{p} \leq \lambda_{m}^{p}
$$

and

$$
\frac{1}{\left(\eta r_{m}\right)^{n-p}} \int_{B_{\eta r_{m}}\left(y_{m}\right)}|D u|^{p} \geq \frac{1}{4} \lambda_{m}^{p}
$$

We rescale our variables to the unit ball $B_{1}(0) \subset \mathbf{R}^{n}$, as follows. For $z \in B_{1}(0)$,

$$
v_{m}(z)=\frac{u\left(r_{m} z+y_{m}\right)-a_{m}}{\lambda_{m}},
$$

where

$$
a_{m}=(u)_{y_{m}, r_{m}}=\frac{1}{\left|B_{r_{m}}\left(y_{m}\right)\right|} \int_{B_{r_{m}}\left(y_{m}\right)} u d x
$$

denotes the average of $u$ over $B_{r_{m}}\left(y_{m}\right)$. Using (3.6), (3.7) and (3.8) we verify that

$$
\sup _{m} \int_{B_{1}(0)}\left|v_{m}\right|^{p} d z<\infty, \quad \sup _{m} \int_{B_{1}(0)}\left|D v_{m}\right|^{p} d z \leq 1,
$$

but

$$
\sup _{m} \frac{1}{\eta^{n-p}} \int_{B_{\eta}(0)}\left|D v_{m}\right|^{p} d z \geq \frac{1}{4} \quad(m=1,2, \ldots)
$$


The sequence $\left\{v_{m}\right\}_{m=1}^{\infty}$ is bounded in $W^{1, p}\left(B_{1}(0), \mathbf{R}^{k}\right)$, and hence there exists a subsequence (which we reindex as necessary and denote also by $\left\{v_{m}\right\}_{m=1}^{\infty}$ ) such that

$$
\begin{gathered}
v_{m} \rightarrow v \text { strongly in } L^{p}\left(B_{1}(0), \mathbf{R}^{k}\right), \\
D v_{m} \rightarrow D v \text { weakly in } L^{p}\left(B_{1}(0), M^{k \times n}\right),
\end{gathered}
$$

$M^{k \times n}$ being the space of the real $k \times n$ matrices.

Next select any smooth function $w: B_{1}(0) \rightarrow \mathbf{R}^{k}$ with compact support. Define

$$
w_{m}(y)=w\left(\frac{y-y_{m}}{r_{m}}\right) \quad \text { for } y \in B_{r_{m}}\left(y_{m}\right)
$$

Since $u$ is weakly $p$-harmonic, (1.2) gives

$$
\int_{B_{r_{m}}\left(y_{m}\right)}|D u|^{p-2} D u: D w_{m} d y=\int_{B_{r_{m}}\left(y_{m}\right)}|D u|^{p-2} A(u)(D u, D u) w_{m} d y .
$$

We rescale this identity to the unit ball, and use the fact that the second fundamental form is a bilinear form in order to obtain the equality

$$
\begin{aligned}
& \int_{B_{1}(0)}\left|D v_{m}\right|^{p-2} D v_{m}: D w d z \\
= & \lambda_{m} \int_{B_{1}(0)}\left|D v_{m}\right|^{p-2} A\left(\lambda_{m} v_{m}+a_{m}\right)\left(D v_{m}, D v_{m}\right) w d z .
\end{aligned}
$$

Thus since $N$ is a smooth compact manifold using (3.9) we have

$$
\left.\left|\int_{B_{1}(0)}\right| D v_{m}\right|^{p-2} D v_{m}:\left.D w d z\left|\leq C \lambda_{m} \int_{B_{1}(0)}\right| D v_{m}\right|^{p} d z \leq C \lambda_{m} .
$$

Note that for each $m=1,2, \ldots, v_{m}$ satisfies the equation

$$
-\operatorname{div}\left(\left|D v_{m}\right|^{p-2} D v_{m}\right)=f_{m} \text { weakly in } B_{1}(0),
$$

where

$$
f_{m}=\lambda_{m}\left|D v_{m}\right|^{p-2} A\left(\lambda_{m} v_{m}+a_{m}\right)\left(D v_{m}, D v_{m}\right),
$$

and

$$
\left\|f_{m}\right\|_{L^{1}\left(B_{1}(0), \mathbf{R}^{k}\right)} \leq C \lambda_{m} \int_{B_{1}(0)}\left|D v_{m}\right|^{p} d z
$$


Hence there exists a further subsequence (which we reindex as necessary and denote also by $\left\{v_{m}\right\}_{m=1}^{\infty}$ ) such that $D v_{m} \rightarrow D v$ a.e. in $\Omega$ (see [HLM]) and

$$
\left|D v_{m}\right|^{p-2} D v_{m} \rightarrow|D v|^{p-2} D v \text { weakly in } L^{p^{\prime}}\left(B_{1}(0), M^{k \times n}\right) .
$$

Letting $m$ go to infinity in (3.13) from (3.14), we deduce

$$
\int_{B_{1}(0)}|D v|^{p-2} D v: D w d z=0 .
$$

This equality is obtained for all $w$ as above. Thus

$$
\operatorname{div}\left(|D v|^{p-2} D v\right)=0 \quad \text { weakly in } B_{1}(0) .
$$

Hence $v \in C_{\mathrm{loc}}^{1, \alpha}(\Omega)$ (see [U], and [E2] for $p \geq 2$; see [T] for $1<p<2$ ) and

$$
\|D v\|_{L^{\infty}\left(B_{1 / 2}(0), M^{k \times n}\right)} \leq C\left(p, n,\|v\|_{W^{1, p}\left(\Omega, \mathbf{R}^{k}\right)}\right)<\infty .
$$

In particular,

$$
\frac{1}{\eta^{n-p}} \int_{B_{\eta}(0)}|D v|^{p} d z \leq C \eta^{p}<\frac{1}{4}
$$

provided that $\eta \in\left(0, \frac{1}{4}\right)$ is chosen small enough.

Next we use Lemma 4.2, to be proved in Section 4. This asserts that

$$
D v_{m} \rightarrow D v \text { strongly in } L^{p}\left(B_{1 / 4}(0), M^{k \times n}\right) .
$$

But then (3.10) forces

$$
\frac{1}{\eta^{n-p}} \int_{B_{\eta}(0)}|D v|^{p} d z \geq \frac{1}{4}
$$

a contradiction to (3.16).

So far it is not clear where the hypothesis that $N$ is a homogeneous space with a left invariant metric is needed. In fact the proof of the strong convergence (3.17) depends heavily on the the structure of the target manifold. We finish this section by giving a characterization of the regular set for a weakly $p$-harmonic map.

Corollary 3.2. Let $u \in W^{1, p}(\Omega, N)$ be a weakly p-harmonic map, and assume that $N\left(x_{0}, u\right)<\varepsilon_{0}, x_{0} \in \Omega$ with $\varepsilon_{0}>0$ as in the decay lemma. Then $u$ is $C^{1, \alpha}$ in a neighborhood of $x_{0}$, for some $\alpha \in(0,1)$. 
Proof. Since $N\left(x_{0}, u\right)<\varepsilon_{0}$ there exists $\rho_{0}>0$ such that $B_{2 \rho_{0}}\left(x_{0}\right) \subset \Omega$, and $N\left(x_{0}, 2 \rho_{0}, u\right) \leq \varepsilon_{0}$. Then for $x \in B_{\rho_{0}}\left(x_{0}\right), N\left(x, \rho_{0}, u\right) \leq N\left(x_{0}, 2 \rho_{0}, u\right) \leq \varepsilon_{0}$, and for $k \geq 1$

$$
N\left(x, \tau^{k} \rho_{0}, u\right) \leq \frac{1}{2^{k}} N\left(x, \rho_{0}, u\right) .
$$

We conclude that for $0<\rho \leq \tau \rho_{0}$,

$$
\frac{1}{\rho^{n-p}} \int_{B_{\rho}(x)}|D u|^{p} \leq N(x, \rho, u) \leq C \rho^{\gamma},
$$

for some $\gamma>0$ and $C>0$. It follows that $u$ is uniformly Hölder continuous on $B_{\rho_{0}}\left(x_{0}\right)$ (see $[\mathrm{GT}]$ ), and therefore $u \in C^{1, \alpha}\left(B_{\rho_{0}}\left(x_{0}\right), N\right)$ for some $\alpha \in(0,1)$ (see $[\mathrm{D}])$.

Corollary 3.3. Let $u \in W^{1, p}(\Omega, N)$ be a weakly p-harmonic map, then $u \in C^{1, \alpha}(\Omega \backslash \mathcal{S}, N)$, where $\mathcal{S}$ is the closed set defined by

$$
\mathcal{S}=\left\{x \in \Omega: N(x, u) \geq \varepsilon_{0}\right\},
$$

where $\varepsilon_{0}>0$ is as in the decay lemma.

Proof. The regularity is a straight forward consequence of Corollary 3.2. In order to prove that $\mathcal{S}$ is closed it is enough to prove that $N(x, u)$ is an upper semi-continuous function of $x$. Let $\left\{x_{i}\right\} \subset \Omega$ be a sequence converging to $x \in \Omega$. Since $B_{\rho}\left(x_{i}\right) \subset B_{\rho+\left|x-x_{i}\right|}(x)$, as long as $B_{\rho+\left|x-x_{i}\right|}(x) \subset \Omega$ we have

$$
N\left(x_{i}, \rho, u\right) \leq N\left(x, \rho+\left|x-x_{i}\right|, u\right) .
$$

For $\varepsilon>0$ there exist $\rho>0$ and $i_{0} \geq 1$ such that

$$
N(x, 2 \rho, u)<N(x, u)+\varepsilon,
$$

and for all $i \geq i_{0},\left|x_{i}-x\right|<\rho$. Hence for $i \geq i_{0}$,

$$
N\left(x_{i}, u\right) \leq N\left(x_{i}, \rho, u\right) \leq N(x, 2 \rho, u)<N(x, u)+\varepsilon .
$$

We conclude that

$$
\lim \sup _{i \rightarrow \infty} N\left(x_{i}, u\right) \leq N(x, u) .
$$

Remark 3.4. Unfortunately, in the general case it is impossible to estimate the size of $\mathcal{R}=\Omega \backslash \mathcal{S}$. As shown by Riviére $[\mathrm{R}], \mathcal{R}$ may be empty, at least in the case $p=2$. In Section 5 we estimate the Hausdorff dimension of $\mathcal{S}$ for stationary $p$-harmonic maps, and for the weak limit of a sequence of stationary $p$-harmonic maps. 
Corollary 3.5. Suppose that $N$ is as above. Let $u \in W^{1, n}(\Omega, N)$ be a weakly p-harmonic map. Then $u \in C^{1, \alpha}(\Omega, N)$ for some $\alpha \in(0,1)$.

Proof. For $B_{r}(y) \subset B_{\rho}(x) \subset \Omega$,

$$
\frac{1}{r^{n-p}} \int_{B_{r}(y)}|D u|^{p} \leq C\left(\int_{B_{r}(y)}|D u|^{n}\right)^{p / n} \leq C\left(\int_{B_{\rho}(x)}|D u|^{n}\right)^{p / n},
$$

and for any $x \in \Omega$

$$
N(x, u) \leq C \lim _{\rho \rightarrow 0}\left(\int_{B_{\rho}(x)}|D u|^{n}\right)^{p / n}=0 .
$$

Therefore $\mathcal{S}=\varnothing$ and $u \in C^{1, \alpha}(\Omega, N)$.

4. Strong Convergence. All the calculations and assertions in Section 3 are routine, except for the compactness assertion (3.17), which depends crucially on the structure of the target manifold. We proceed to establish that result now.

Let $\vartheta: \mathbf{R}^{n} \rightarrow \mathbf{R}$ be a smooth cutoff function satisfying

$$
0 \leq \vartheta \leq 1, \quad \vartheta=1 \text { on } B_{1 / 4}(0), \quad \vartheta=0 \text { on } \mathbf{R}^{n} \backslash B_{3 / 8}(0)
$$

Lemma 4.1. The sequence $\left\{\vartheta v_{m}\right\}_{m \geq 1}$ is bounded in $\mathrm{BMO}\left(\mathbf{R}^{n}, \mathbf{R}^{k}\right)$.

Proof. We claim that for each $m=1,2, \ldots$,

$$
\begin{aligned}
\left\|\vartheta v_{m}\right\|_{*} & \leq C \sup \left\{\frac{1}{r^{n-1}} \int_{B_{r}(x)}\left|D v_{m}\right| d x: B_{r}(x) \subset B_{1 / 2}(0)\right\} \\
& \leq C N\left(0, \frac{1}{2}, v_{m}\right)^{1 / p} \leq C N\left(0,1, v_{m}\right)^{1 / p}
\end{aligned}
$$

For a proof of this statment see either [E1] or [B1]. Note that if $B_{r}(x) \subset B_{1}(0)$, then $B_{r r_{m}}\left(r_{m} x+y_{m}\right) \subset B_{r_{m}}\left(y_{m}\right)$. Since

$$
N\left(0,1, v_{m}\right)=\sup \left\{\frac{1}{r^{n-p}} \int_{B_{r}(x)}\left|D v_{m}\right|^{p}: B_{r}(x) \subset B_{1}(0)\right\},
$$

and

$$
\frac{1}{r^{n-p}} \int_{B_{r}(x)}\left|D v_{m}\right|^{p}=\frac{1}{\lambda_{m}^{p}} \cdot \frac{1}{\left(r_{m} r\right)^{n-p}} \int_{B_{r_{m}}\left(r_{m} x+y_{m}\right)}|D u|^{p},
$$


we conclude that

$$
N\left(0,1, v_{m}\right) \leq \frac{1}{\lambda_{m}^{p}} N\left(y_{m}, r_{m}, u\right) .
$$

Recall that $B_{r_{m}}\left(y_{m}\right) \subset B_{\rho_{m} / 2}\left(x_{m}\right) \subset B_{\rho_{m}}\left(x_{m}\right)$, and therefore

$$
N\left(0,1, v_{m}\right) \leq \frac{1}{\lambda_{m}^{p}} N\left(x_{m}, \rho_{m}, u\right)=1 .
$$

Lemma 4.2. The rescaled functions $\left\{D v_{m}\right\}_{m \geq 1}$ converge strongly to Dv in $L^{p}\left(B_{1 / 4}(0), M^{k \times n}\right)$.

Proof. Since $u$ satisfies equation (2.2), then

(4.1) $\operatorname{div}\left(\left|D v_{m}\right|^{p-2} D v_{m}\right)$

$$
=\lambda_{m} \sum_{\beta=1}^{n} \sum_{i=1}^{q}\left|D v_{m}\right|^{p-2}\left\langle D_{\beta} v_{m}, X_{i}\left(\lambda_{m} v_{m}+a_{m}\right)\right\rangle\left(\frac{1}{\lambda_{m}} D_{\beta} Y_{i}\left(\lambda_{m} v_{m}+a_{m}\right)\right)
$$

weakly in $B_{1}(0)$ for each $m=1,2, \ldots$. Since

$$
\operatorname{div}\left(\sum_{i}^{q}|D u|^{p-2}\left\langle D u, X_{i}(u)\right\rangle\right)=\sum_{\alpha}^{n} \sum_{i}^{q} D_{\alpha}\left(|D u|^{p-2}\left\langle D_{\alpha} u, X_{i}(u)\right\rangle\right)=0
$$

weakly in $\Omega$, a simple change of variables shows that

$$
\begin{aligned}
& \operatorname{div}\left(\sum_{i=1}^{q}\left|D v_{m}\right|^{p-2}\left\langle D v_{m}, X_{i}\left(\lambda_{m} v_{m}+a_{m}\right)\right\rangle\right) \\
= & \sum_{\beta=1}^{n} \sum_{i=1}^{q} D_{\beta}\left(\left|D v_{m}\right|^{p-2}\left\langle D_{\beta} v_{m}, X_{i}\left(\lambda_{m} v_{m}+a_{m}\right)\right\rangle\right)=0
\end{aligned}
$$

weakly in $B_{1}(0)$. Therefore, applying the Proposition from [CLMS] we conclude that

$$
\begin{array}{r}
\left.\sum_{\beta=1}^{n} \sum_{i \underline{\underline{m}}}^{q}(y \Phi)_{\boldsymbol{y}_{m}}\right|^{p-2}\left\langle D_{\beta} v_{m}(y), X_{i}\left(\lambda_{m} v_{m}(y)+a_{m}\right)\right\rangle \\
\cdot\left(\frac{1}{\lambda_{m}} D_{\beta} Y_{i}\left(\lambda_{m} v_{m}+a_{m}\right)\right)
\end{array}
$$

belongs to $\mathcal{H}_{\text {loc }}^{1}\left(B_{1}(0)\right)$, and

$$
\sup _{m \geq 1}\left\|h_{m}\right\|_{L^{1}\left(B_{1}(0)\right)} \leq C \sup _{m \geq 1} \int_{B_{1}(0)}\left|D v_{m}\right|^{p} d x \leq C .
$$


For a compact set $K \subset B_{1}(0)$ we estimate $\sup _{m \geq 1}\left\|h_{m}\right\|_{\mathcal{H}^{1}(K)}$ using (2.6) and (3.9),

$$
\begin{gathered}
\sup _{m \geq 1}\left\|h_{m}\right\|_{\mathcal{H}^{1}(K)} \\
\leq C\left\|\sum_{i=1}^{q}\left|D v_{m}\right|^{p-2}\left\langle D v_{m}(y), X_{i}\left(\lambda_{m} v_{m}(y)+a_{m}\right)\right\rangle\right\|_{L^{p^{\prime}\left(B_{1}(0), \mathbf{R}^{n}\right)}}^{p^{\prime}} \\
\quad+C\left\|\sum_{i}^{q}\left(\frac{1}{\lambda_{m}} D Y_{i}\left(\lambda_{m} v_{m}+a_{m}\right)\right)\right\|_{L^{p}\left(B_{1}(0), \mathbf{R}^{n}\right)}^{p}, \\
\sup _{m \geq 1}\left\|h_{m}\right\|_{\mathcal{H}^{1}(K)} \leq C\left\|D v_{m}\right\|_{L^{p}\left(B_{1}(0), \mathbf{R}^{n}\right)}^{p} \leq C .
\end{gathered}
$$

Note that $\int \vartheta \neq 0$. Hence for each $m=1,2, \ldots, \vartheta\left(h_{m}-\mu_{m}\right) \in \mathcal{H}^{1}\left(\mathbf{R}^{n}\right)$, where

$$
\mu_{m}=\frac{\int h_{m} \vartheta}{\int \vartheta} \text { and } \sup _{m \geq 1}\left\|\vartheta\left(h_{m}-\mu_{m}\right)\right\|_{\mathcal{H}^{1}\left(\mathbf{R}^{n}\right)} \leq C .
$$

Since

$$
\operatorname{div}\left(\left|D v_{m}\right|^{p-2} D v_{m}\right)=\lambda_{m} h_{m} \quad \text { weakly in } B_{1}(0)
$$

and

$$
\operatorname{div}\left(|D v|^{p-2} D v\right)=0 \quad \text { weakly in } B_{1}(0),
$$

then for any $w \in W_{0}^{1, p}\left(B_{1}(0), \mathbf{R}^{k}\right) \cap L^{\infty}\left(B_{1}(0), \mathbf{R}^{k}\right)$ we have

$$
\int_{B_{1}(0)}\left(|D v|^{p-2} D v-\left|D v_{m}\right|^{p-2} D v_{m}\right): D w d x=\lambda_{m} \int_{B_{1}(0)} h_{m} w d x
$$

We now insert $w=\vartheta^{2}\left(v-v_{m}\right)$ into (4.5),

$$
\begin{aligned}
& \int_{B_{1}(0)} \vartheta^{2}\left(|D v|^{p-2} D v-\left|D v_{m}\right|^{p-2} D v_{m}\right):\left(D v-D v_{m}\right) d x \\
& +2 \int_{B_{1}(0)} \vartheta\left(|D v|^{p-2} D v-\left|D v_{m}\right|^{p-2} D v_{m}\right):\left(D \vartheta \otimes\left(v-v_{m}\right)\right) d x \\
& =\lambda_{m} \int_{B_{1}(0)} h_{m} \vartheta^{2}\left(v-v_{m}\right) d x .
\end{aligned}
$$


For $a, b \in \mathbf{R}^{\ell}$ and $p \geq 2$,

(4.7) $|a-b|^{p} \leq 2^{p-2}\left(|a|^{p-2}+|b|^{p-2}\right)|a-b|^{2} \leq 2^{p-1}\left(|a|^{p-2} a-|b|^{p-2} b\right) \cdot(a-b)$.

Applying (4.7)

$$
\begin{gathered}
\int_{B_{1}(0)} \vartheta^{2}\left|D v-D v_{m}\right|^{p} d x \\
\leq C \int_{B_{1}(0)} \vartheta\left|\left(|D v|^{p-2} D v-\left|D v_{m}\right|^{p-2} D v_{m}\right)\right||D \vartheta|\left|v-v_{m}\right| d x \\
+C \lambda_{m}\left|\int_{B_{1}(0)} h_{m} \vartheta^{2}\left(v-v_{m}\right) d x\right|
\end{gathered}
$$

For $a, b \in \mathbf{R}^{\ell}$ and $1<p<2($ see $[\mathrm{HLM}])$

$$
\left(|a|^{p-2} a-|b|^{p-2} b\right) \cdot(a-b) \geq(p-1)(|a|+|b|)^{p-2}|a-b|^{2} .
$$

Applying (4.9) as in Corollary 2 in [HLM], (3.9), (3.15) and (4.6) we have

$$
\int_{B_{1}(0)} \vartheta^{2}\left|D v-D v_{m}\right|^{p} d x
$$$$
\leq\left(\int_{B_{1}(0)} \vartheta^{2}\left(|D v|+\left|D v_{m}\right|\right)^{p} d x\right)^{(2-p) / 2}
$$

$$
\cdot\left(\frac{1}{p-1} \int_{B_{1}(0)} \vartheta^{2}\left(|D v|^{p-2} D v-\left|D v_{m}\right|^{p-2} D v_{m}\right): D\left(v-v_{m}\right) d x\right)^{p / 2}
$$$$
\leq C\left(\int_{B_{1}(0)} \vartheta\left|\left(|D v|^{p-2} D v-\left|D v_{m}\right|^{p-2} D v_{m}\right)\right||D \vartheta|\left|v-v_{m}\right| d x\right)^{p / 2}
$$

$$
+C\left(\lambda_{m}\left|\int_{B_{1}(0)} h_{m} \vartheta^{2}\left(v-v_{m}\right) d x\right|\right)^{p / 2} .
$$

In both cases we have similar terms, we consider each of them separately. Applying (3.9) and Hölder's inequality, we estimate the first term as follows

$$
(4.11) \int_{B_{1}(0)} \vartheta\left|\left(|D v|^{p-2} D v-\left|D v_{m}\right|^{p-2} D v_{m}\right)\right||D \vartheta|\left|v-v_{m}\right| d x
$$




$$
\begin{aligned}
& \leq\left(\int_{B_{1}(0)} \vartheta^{p^{\prime}}\left(|D v|^{p-1}+\left|D v_{m}\right|^{p-1}\right)^{p^{\prime}} d x\right)^{1 / p^{\prime}}\left(\int_{B_{1}(0)}|D \vartheta|^{p}\left|v-v_{m}\right|^{p} d x\right)^{1 / p} \\
& \leq C\left(\int_{B_{1}(0)}\left(|D v|^{p}+\left|D v_{m}\right|^{p}\right) d x\right)^{1 / p^{\prime}}\left(\int_{B_{1}(0)}\left|v-v_{m}\right|^{p} d x\right)^{1 / p} \\
& \leq C\left(\int_{B_{1}(0)}\left|v-v_{m}\right|^{p} d x\right)^{1 / p} .
\end{aligned}
$$

Note that

$$
\begin{array}{r}
\int_{B_{1}(0)} h_{m} \vartheta^{2}\left(v-v_{m}\right) d x=\int_{B_{1}(0)} \vartheta\left(h_{m}-\mu_{m}\right) \vartheta\left(v-v_{m}\right) d x \\
+\int_{B_{1}(0)} \mu_{m} \vartheta^{2}\left(v-v_{m}\right) d x
\end{array}
$$

and

$$
\begin{aligned}
\left|\int_{B_{1}(0)} \mu_{m} \vartheta^{2}\left(v-v_{m}\right) d x\right| & \leq\left|\mu_{m}\right|\left(\int_{\mathbf{R}^{n}} \vartheta\right) \int_{B_{1}(0)}\left|v-v_{m}\right| d x \\
& \leq C\left(\int_{B_{1}(0)}\left|h_{m}\right| d x\right)\left(\int_{B_{1}(0)}\left|v-v_{m}\right| d x\right) \\
& \leq C\left(\int_{B_{1}(0)}\left|D v_{m}\right|^{p} d x\right)\left(\int_{B_{1}(0)}\left|v-v_{m}\right| d x\right), \\
\left|\int_{B_{1}(0)} \mu_{m} \vartheta^{2}\left(v-v_{m}\right) d x\right| & \leq C \int_{B_{1}(0)}\left|v-v_{m}\right| d x .
\end{aligned}
$$

In order to estimate $\int_{B_{1}(0)} \vartheta\left(h_{m}-\mu_{m}\right) \vartheta\left(v-v_{m}\right) d x$, we use the duality between $\mathcal{H}^{1}\left(\mathbf{R}^{n}\right)$ and BMO. Note that since $v$ is weakly $p$-harmonic in $B_{1}(0)$, $\|D v\|_{L^{\infty}\left(B_{1 / 2}(0), M^{k \times n}\right)}$ is bounded, see (3.15). Thus

$$
\|\vartheta v\|_{*} \leq C N\left(0, \frac{1}{2}, v\right)^{1 / p} \leq C .
$$

Using Lemma 4.1, and inequality (2.3) we have

$$
\begin{aligned}
& \left|\int_{B_{1}(0)} \vartheta\left(h_{m}-\mu_{m}\right) \vartheta\left(v-v_{m}\right) d x\right| \\
\leq & \left\|\vartheta\left(h_{m}-\mu_{m}\right)\right\|_{\mathcal{H}^{1}\left(\mathbf{R}^{n}\right)}\left\|\vartheta\left(v-v_{m}\right)\right\|_{*} \\
\leq & C\left(\|\vartheta v\|_{*}+\left\|\vartheta v_{m}\right\|_{*}\right) \leq C .
\end{aligned}
$$


Putting together (4.8), (4.10), (4.11), (4.12), (4.13) and (4.14) we have for $2 \leq$ $p \leq n$

$$
\begin{aligned}
& \int_{B_{1 / 4}(0)}\left|D v-D v_{m}\right|^{p} d x \\
\leq & \int_{B_{1}(0)} \vartheta^{2}\left|D v-D v_{m}\right|^{p} d x \\
\leq & C\left(\int_{B_{1}(0)}\left|v-v_{m}\right|^{p} d x\right)^{1 / p}+C \lambda_{m}\left(1+\int_{B_{1}(0)}\left|v-v_{m}\right| d x\right),
\end{aligned}
$$

and for $1<p<2$,

$$
\begin{aligned}
& \int_{B_{1 / 4}(0)}\left|D v-D v_{m}\right|^{p} d x \\
\leq & \int_{B_{1}(0)} \vartheta^{2}\left|D v-D v_{m}\right|^{p} d x \\
\leq & C\left(\int_{B_{1}(0)}\left|v-v_{m}\right|^{p} d x\right)^{1 / 2}+C \lambda_{m}^{p / 2}\left(1+\int_{B_{1}(0)}\left|v-v_{m}\right| d x\right)^{p / 2} .
\end{aligned}
$$

Since $v_{m} \rightarrow v$ strongly in $L^{p}\left(B_{1}(0), \mathbf{R}^{k}\right)$, and $\lambda_{m} \rightarrow 0$, letting $m$ go to infinity in (4.15) and (4.16) we conclude that for $1<p \leq n$,

$$
\int_{B_{1 / 4}(0)}\left|D v-D v_{m}\right|^{p} d x \rightarrow 0
$$

i.e., $D v_{m} \rightarrow D v$ strongly in $L^{p}\left(B_{1 / 4}(0), M^{k \times n}\right)$.

5. Regularity Properties for some Weakly $p$-Harmonic Maps. Now we show that under the hypothesis of Theorems 2 and 3, it is possible to estimate the size of the set $\mathcal{S}$ defined in Corollary 3.3.

Theorem 2. Let $(N, h)$ be a smooth compact homogeneous space, and $h$ a left invariant metric on $N$. Assume that $u \in W^{1, p}(\Omega, N)$ is a stationary p-harmonic map. Then there exists a closed subset $\mathcal{S} \subset \Omega$ such that

$$
\mathcal{H}^{n-p}(\mathcal{S})=0, \quad u \in C^{1, \alpha}(\Omega \backslash \mathcal{S}, N),
$$

for some $\alpha \in(0,1)$. Here $\mathcal{H}^{n-p}$ denotes the $(n-p)$-dimensional Hausdorff measure. 
Proof. Recall that a stationary $p$-harmonic map $u$ satisfies the monotonicity inequalities, namely

$$
\frac{1}{r^{n-p}} \int_{B_{r}(y)}|D u|^{p} d y \leq \frac{1}{\rho^{n-p}} \int_{B_{\rho}(y)}|D u|^{p} d y,
$$

for all concentric balls $B_{r}(y) \subset B_{\rho}(y) \subset \Omega$, and

$$
\lim _{\rho \rightarrow 0} \frac{1}{\rho^{n-p}} \int_{B_{\rho}(y)}|D u|^{p} d y \text { exists. }
$$

Moreover if $B_{r}(y) \subset B_{\rho}(x) \subset \Omega$, then

$$
\frac{1}{\rho^{n-p}} \int_{B_{\rho}(y)}|D u|^{p} d y \leq 2^{n-p} \frac{1}{(2 \rho)^{n-p}} \int_{B_{2}(x)}|D u|^{p} d y .
$$

Putting together (5.1) and (5.2), and taking the supremum over all balls $B_{r}(y) \subset$ $B_{\rho}(x)$, we obtain

$$
N(x, \rho, u) \leq 2^{n-p} \frac{1}{(2 \rho)^{n-p}} \int_{B_{2 \rho}(x)}|D u|^{p} d y .
$$

Letting $\rho$ go to zero in (5.3)

$$
N(x, u) \leq 2^{n-p} \lim _{\rho \rightarrow 0} \frac{1}{\rho^{n-p}} \int_{B_{\rho}(x)}|D u|^{p} d y
$$

Hence for $\mathcal{S}$ defined as in Corollary 3.3,

$$
\mathcal{S}=\left\{x \in \Omega: N(x, u) \geq \varepsilon_{0}\right\} \subset\left\{x \in \Omega: \lim _{\rho \rightarrow 0} \frac{1}{\rho^{n-p}} \int_{B_{\rho}(x)}|D u|^{p} d y \geq \frac{\varepsilon_{0}}{2^{n-p}}\right\}
$$

but

$$
\mathcal{H}^{n-p}\left(\left\{x \in \Omega: \lim _{\rho \rightarrow 0} \frac{1}{\rho^{n-p}} \int_{B_{\rho}(x)}|D u|^{p} d y>0\right\}\right)=0
$$

see [EG], Section 2.4.3. Then

$$
\mathcal{H}^{n-p}(\mathcal{S})=0
$$

and $u \in C^{1, \alpha}(\Omega \backslash \mathcal{S}, N)$, for some $\alpha \in(0,1)$. 
Theorem 3. Let $(N, h)$ be a smooth compact homogeneous space, and $h$ a left invariant metric on $N$. Let $u_{k} \in W^{1, p}(\Omega, N)$ be a sequence of stationary $p$-harmonic maps, and assume that $u_{k} \rightarrow u$ in $W^{1, p}(\Omega, N)$. Then there exists a closed subset $\mathcal{S} \subset \Omega$ such that

$$
u \in C^{1, \alpha}(\Omega \backslash \mathcal{S}, N),
$$

for some $\alpha \in(0,1)$, and for any compact set $K \subset \Omega$

$$
\mathcal{H}^{n-p}(\mathcal{S} \cap K)<\infty .
$$

Proof. Let $\mathcal{S}=\left\{x \in \Omega: N(x, u) \geq \varepsilon_{0}\right\}$ be as in Corollary 3.3, and let $K$ be a compact set in $\Omega$. Since $\mathcal{S}$ is closed then $\mathcal{S} \cap K$ is compact. Let $\delta>0$, cover $\mathcal{S} \cap K$ by ball $B_{\rho_{i}}\left(x_{i}\right)$ centered on $\mathcal{S}$, and so that $\rho_{i} \leq \delta$. Since $\mathcal{S} \cap K$ is compact, there is a disjoint finite subcollection $\left\{B_{\rho_{i}}\left(x_{i}\right)\right\}_{1 \leq i \leq N}$ such that $\mathcal{S} \cap K \subset \bigcup_{i=1}^{N} B_{5 \rho_{i}}\left(x_{i}\right)$. But since $x_{i} \in \mathcal{S}, N\left(x_{i}, u\right) \geq \varepsilon_{0}$, there exist $y_{i} \in B_{\rho_{i} / 2}\left(x_{i}\right)$ and $r_{i} \in\left(0, \rho_{i} / 2\right)$ such that

$$
\frac{\varepsilon_{0}}{2} \leq \frac{1}{r_{i}^{n-p}} \int_{B_{r_{i}}\left(y_{i}\right)}|D u|^{p} \leq \frac{1}{r_{i}^{n-p}} \liminf _{k \rightarrow \infty} \int_{B_{r_{i}}\left(y_{i}\right)}\left|D u_{k}\right|^{p},
$$

where in order to obtain the second inequality we have used the lower semicontinuity of the $L^{p}$ norm. There exists $k_{i} \in \mathbf{N}$ such that $\forall k \geq k_{i}$,

$$
\frac{\varepsilon_{0}}{4} \leq \frac{1}{2} \cdot \frac{1}{r_{i}^{n-p}} \int_{B_{r_{i}}\left(y_{i}\right)}|D u|^{p} \leq \frac{1}{r_{i}^{n-p}} \int_{B_{r_{i}}\left(y_{i}\right)}\left|D u_{k}\right|^{p},
$$

and by applying the monotonicity inequality to each $u_{k}$, we have

$$
\begin{aligned}
\frac{\varepsilon_{0}}{4} \leq \frac{1}{r_{i}^{n-p}} \int_{B_{r_{i}}\left(y_{i}\right)}\left|D u_{k}\right|^{p} & \leq\left(\frac{2}{\rho_{i}}\right)^{n-p} \int_{B_{\rho_{i} / 2}\left(y_{i}\right)}\left|D u_{k}\right|^{p} \\
& \leq \frac{2^{n-p}}{\rho_{i}^{n-p}} \int_{B_{\rho_{i}}\left(x_{i}\right)}\left|D u_{k}\right|^{p} .
\end{aligned}
$$

Thus, since the sequence $u_{k}$ is uniformly bounded in $W^{1, p}(\Omega, N)$, for $k \geq$ $\max \left\{k_{i}: 1 \leq i \leq N\right\}$,

$$
\sum_{i}^{N} \rho_{i}^{n-p} \leq C\left(n, \varepsilon_{0}\right) \int_{\cup_{i=1}^{N} B_{\rho_{i}}\left(x_{i}\right)}\left|D u_{k}\right|^{p} \leq C \int_{\Omega}\left|D u_{k}\right|^{p} \leq C,
$$

and for $\delta>0$,

$$
\mathcal{H}_{5 \delta}^{n-p}(\mathcal{S} \cap K) \leq C \sum_{i}^{N}\left(5 \rho_{i}\right)^{n-p} \leq C
$$

which implies $\mathcal{H}^{n-p}(\mathcal{S} \cap K) \leq C\left(n, \varepsilon_{0}\right)$. 
Theorem 4. Let $M$ be a smooth compact manifold. Let $\Omega$ be an open set in $\mathbf{R}^{n}$ for $n \geq 3$, let $u_{k} \in W^{1,2}(\Omega, M)$ be a sequence of stationary harmonic maps, and assume that $u_{k} \rightarrow u$ in $W^{1,2}(\Omega, M)$. Then $u$ is a harmonic map, and there exists a closed subset $\Sigma \subset \Omega$ such that

$$
u \in C^{\infty}(\Omega \backslash \Sigma, M)
$$

and for any compact set $K \subset \Omega$

$$
\mathcal{H}^{n-2}(\Sigma \cap K)<\infty
$$

Let us recall a result of Bethuel's [B1, Theorem I.4],

Theorem ([B1]). There exists $\varepsilon_{1}>0$ such that is $v \in W^{1,2}(\Omega, M)$ is a stationary harmonic map, and for $B_{r_{0}}\left(x_{0}\right) \subset \Omega$, v satisfies

$$
\frac{1}{r_{0}^{n-2}} \int_{B_{r_{0}}\left(x_{0}\right)}|D v|^{2}<\varepsilon_{1}^{2}
$$

then $v$ is smooth on $B_{r_{0} / 2}\left(x_{0}\right)$. Moreover, for $\ell \geq 1$,

$$
\|v\|_{C^{\ell}\left(B_{r_{0} / 2}\left(x_{0}\right), M\right)} \leq C\left(\varepsilon_{1}, n, k, \ell\right) .
$$

Proof of Theorem 4. We define $\Sigma$, as in [Sch], by

$$
\Sigma=\bigcap_{r>0}\left\{x \in \Omega: \liminf _{k \rightarrow \infty} \frac{1}{r^{n-2}} \int_{B_{r}(x)}\left|D u_{k}\right|^{2} \geq \varepsilon_{1}^{2}\right\},
$$

where $\varepsilon_{1}>0$ is as in Bethuel's Theorem. We claim that $\Sigma$ is a closed subset of $\Omega$ with locally finite $(n-2)$-dimensional Hausdorff measure, and that $u \in$ $C^{\infty}(\Omega \backslash \Sigma, M)$.

In fact, let $\left\{x_{i}\right\}$ be a sequence in $\Sigma$ and assume $x_{i} \rightarrow x \in \Omega$. Suppose $x \notin \Sigma$, there exists $r>0$ such that

$$
\liminf _{k \rightarrow \infty} \frac{1}{r^{n-2}} \int_{B_{r}(x)}\left|D u_{k}\right|^{2}<\varepsilon_{1}^{2}
$$

There exist $\delta>0$, and a subsequence $\left\{u_{n_{k}}\right\}$ (depending on $x, r$ and $\delta$ ) such that for each $n_{k}$

$$
\frac{1}{r^{n-2}} \int_{B_{r}(x)}\left|D u_{n_{k}}\right|^{2}<\varepsilon_{1}^{2}-\delta
$$


Taking $i$ large enough in order to guarantee that $\left|x-x_{i}\right|<r$ and applying the monotonicity inequality (5.1) to each $u_{n_{k}}$ we have

$$
\begin{aligned}
\left(\frac{r}{2}\right)^{2-n} \int_{B_{r / 2}\left(x_{i}\right)}\left|D u_{n_{k}}\right|^{2} & \leq\left(r-\left|x_{i}-x\right|\right)^{2-n} \int_{B_{\left(r-\left|x_{i}-x\right|\right)}\left(x_{i}\right)}\left|D u_{n_{k}}\right|^{2} \\
& \leq\left(r-\left|x_{i}-x\right|\right)^{2-n} \int_{B_{r}(x)}\left|D u_{n_{k}}\right|^{2} \\
& \leq\left(\frac{r}{r-\left|x-x_{i}\right|}\right)^{n-2} \cdot \frac{1}{r^{n-2}} \int_{B_{r}(x)}\left|D u_{n_{k}}\right|^{2} \\
& \leq\left(\frac{r}{r-\left|x-x_{i}\right|}\right)^{n-2}\left(\varepsilon_{1}^{2}-\delta\right) .
\end{aligned}
$$

Choosing $i$ large enough we can insure that for each $n_{k}$

$$
\left(\frac{r}{2}\right)^{2-n} \int_{B_{r / 2}\left(x_{i}\right)}\left|D u_{n_{k}}\right|^{2} \leq\left(\frac{r}{r-\left|x-x_{i}\right|}\right)^{n-2}\left(\varepsilon_{1}^{2}-\delta\right)<\varepsilon_{1}^{2},
$$

which contradicts the fact that $x_{i} \in \Sigma$. Therefore $x \in \Sigma$ and $\Sigma$ is a closed subset of $\Omega$.

Let $K \subset \Omega$ be a compact set. For $\delta>0$ there exists a finite collection of disjoint balls $\left\{B_{r_{i}}\left(x_{i}\right)\right\}_{1 \leq i \leq N}$ centered on $\Sigma$, so that $\Sigma \cap K \subset \bigcup_{i=1}^{N} B_{5 r_{i}}\left(x_{i}\right)$, and

$$
\liminf _{k \rightarrow \infty} \frac{1}{r_{i}^{n-2}} \int_{B_{r_{i}}\left(x_{i}\right)}\left|D u_{k}\right|^{2} \geq \varepsilon_{1}^{2} .
$$

Hence for each $i=1, \ldots, N$, there exists a subsequence $\left\{u_{i_{k}}\right\}$ (depending on $x_{i}$, $r_{i}$ and $\left.\varepsilon_{1}\right)$ such that for each $i_{k}$

$$
\frac{1}{r_{i}^{n-2}} \int_{B_{r_{i}}\left(x_{i}\right)}\left|D u_{i_{k}}\right|^{2} \geq \frac{\varepsilon_{1}^{2}}{2}
$$

It is possible by taking successive subsequences to construct a subsequence $\left\{u_{n_{k}}\right\}$ so that for each $i=1, \ldots, N$,

$$
\frac{1}{r_{i}^{n-2}} \int_{B_{r_{i}}\left(x_{i}\right)}\left|D u_{n_{k}}\right|^{2} \geq \frac{\varepsilon_{1}^{2}}{2} .
$$

Then

$$
\begin{aligned}
\mathcal{H}_{5 \delta}^{n-2}(\Sigma \cap K) & \leq C \sum_{i=1}^{N}\left(5 r_{i}\right)^{n-2} \leq C\left(n, \varepsilon_{1}\right) \sum_{i=1}^{N} \int_{B_{r_{i}}\left(x_{i}\right)}\left|D u_{n_{k}}\right|^{2} \\
& \leq C\left(n, \varepsilon_{1}\right) \int_{\cup_{i=1}^{N} B_{r_{i}}\left(x_{i}\right)}\left|D u_{n_{k}}\right|^{2} \leq C\left(n, \varepsilon_{1}\right) \int_{\Omega}\left|D u_{n_{k}}\right|^{2} \leq C,
\end{aligned}
$$


and therefore $\mathcal{H}^{n-2}(\Sigma \cap K) \leq C\left(n, \varepsilon_{1}\right)$.

In order to prove that $u \in C^{\infty}(\Omega \backslash \Sigma, M)$, let $x \notin \Sigma$. There exists $r>0$ such that

$$
\liminf _{k \rightarrow \infty} \frac{1}{r^{n-2}} \int_{B_{r}(x)}\left|D u_{k}\right|^{2}<\varepsilon_{1}^{2} .
$$

There is subsequence $\left\{u_{n_{k}}\right\}$ (depending on $x, r$ and $\varepsilon_{1}$ ) such that for each $n_{k}$

$$
\frac{1}{r^{n-2}} \int_{B_{r}(x)}\left|D u_{n_{k}}\right|^{2}<\varepsilon_{1}^{2}-\delta
$$

Bethuel's result guarantees that $u_{n_{k}} \in C^{\infty}\left(B_{r / 2}(x), M\right)$, and

$$
\left\|u_{n_{k}}\right\|_{C^{\ell}\left(B_{r / 2}(x), M\right)} \leq C\left(\varepsilon_{1}, n, \ell\right) .
$$

Then $u_{n_{k}} \rightarrow u$ in $C^{2}\left(B_{r / 2}(x), M\right)$, and $u$ is a classical harmonic map from $B_{r / 2}(x)$ into $M$. Elliptic regularity theory guarantees that $u \in C^{\infty}\left(B_{r / 2}(x), M\right)$.

In order to prove that $u$ is a harmonic map from $\Omega$ into $M$, we use the fact that a set of finite $(n-2)$-dimensional Hausdorff measure in $\mathbf{R}^{n}$ has 2 capacity zero (see [EG] Section 4.7.2). Let $w \in W^{1,2}\left(\Omega, \mathbf{R}^{k}\right) \cap L^{\infty}\left(\Omega, \mathbf{R}^{k}\right)$ be a test function with compact support. Denote by $K$ the support of $w$, since $\mathcal{H}^{n-2}(\Sigma \cap K)<\infty$, then $\operatorname{Cap}_{2}(\Sigma \cap K)=0$. There exists a sequence $\left\{\eta_{i}\right\}$ of functions in $\mathbf{R}^{n}$ such that $0 \leq \eta_{i} \leq 1$,

$$
\Sigma \cap K \subset \operatorname{int}\left\{\eta_{i}=1\right\}
$$

$$
\int_{\mathbf{R}^{n}}\left|D \eta_{i}\right|^{2} \rightarrow 0 \quad \text { as } i \rightarrow \infty
$$

in particular,

$$
\int_{\mathbf{R}^{n}} \eta_{i}^{2 n /(n-2)} \rightarrow 0 \quad \text { and } \quad \eta_{i} \rightarrow 0 \text { almost everywhere } \mathbf{R}^{n} \text { as } i \rightarrow \infty
$$

Then

$$
\begin{aligned}
\int_{\Omega} D u: D w & =\int_{\Omega} D u: D\left(\left(1-\eta_{i}\right) w+\eta_{i} w\right) \\
& =\int_{\Omega} D u: D\left(\left(1-\eta_{i}\right) w\right)+\int_{\Omega} \eta_{i} D u: D w+\int_{\Omega} D u:\left(D \eta_{i} \otimes w\right) .
\end{aligned}
$$


From (5.4) we conclude that $\left(1-\eta_{i}\right) w$ is compactly supported in $\Omega \backslash \Sigma$, and therefore,

$$
\int_{\Omega} D u: D\left(\left(1-\eta_{i}\right) w\right)=\int_{\Omega} A(u)(D u, D u)\left(1-\eta_{i}\right) w .
$$

By the Dominated Convergence Theorem and (5.6), we have

$$
\begin{gathered}
\int_{\Omega} A(u)(D u, D u) \eta_{i} w \rightarrow 0 \quad \text { as } i \rightarrow \infty, \\
\int_{\Omega} \eta_{i} D u: D w \rightarrow 0 \quad \text { as } i \rightarrow \infty .
\end{gathered}
$$

Applying (5.5) we have

$$
\left|\int_{\Omega} D u:\left(D \eta_{i} \otimes w\right)\right| \leq\|w\|_{L^{\infty}\left(\Omega, \mathbf{R}^{k}\right)}\left(\int_{\Omega}|D u|^{2}\right)^{1 / 2}\left(\int_{\mathbf{R}^{n}}\left|D \eta_{i}\right|^{2}\right)^{1 / 2} \rightarrow 0
$$

as $i \rightarrow \infty$; therefore,

$$
\int_{\Omega} D u: D w=\int_{\Omega} A(u)(D u, D u) w .
$$

Remark 5.1. Let $N$ be a smooth compact homogenous space with a left invariant metric. Let $u_{k} \in W^{1,2}(\Omega, N)$ be a sequence of stationary harmonic maps, and assume that $u_{k} \rightarrow u$ in $W^{1,2}(\Omega, N)$. Then $u$ is a harmonic map. From Theorem 3 we know that $u \in C^{\infty}(\Omega \backslash \mathcal{S}, N)$, where

$$
\mathcal{S}=\left\{x \in \Omega: N(x, u) \geq \varepsilon_{0}\right\},
$$

and from Theorem 4 we know that $u \in C^{\infty}(\Omega \backslash \Sigma, N)$, where

$$
\Sigma=\bigcap_{r>0}\left\{x \in \Omega: \liminf _{k \rightarrow \infty} \frac{1}{r^{n-2}} \int_{B_{r}(x)}\left|D u_{k}\right|^{2} \geq \varepsilon_{1}^{2}\right\} .
$$

Note that $\mathcal{S} \subset \Sigma$. In general these two sets are not equal. There is an example, due to R. Hardt, of a sequence of stationary harmonic maps from $B_{1}(0) \subset \mathbf{R}^{3}$ into $S^{2}$ obtained by rescaling a harmonic map with a singularity at the origin. The limit map is constant and therefore $\mathcal{S}=\varnothing$. On the other hand $\Sigma=\{0\}$.

In some sense $\Sigma$ measures the failure of the strong convergence of $u_{k}$ to $u$ in $W^{1,2}(\Omega, M)$. In fact, if $\Omega^{\prime} \Subset \Omega$ and $\mathcal{H}^{n-2}\left(\Sigma \cap \bar{\Omega}^{\prime}\right)=0$, then $u_{k} \rightarrow u$ strongly in $W^{1,2}\left(\Omega^{\prime}, N\right)$. In particular, we can then assert that $u$ is stationary in $\Omega^{\prime}$. 


\section{REFERENCES}

[B1] F. Bethuel, On the singular set of stationary harmonic maps, Manuscripta Math. 78 (1993), 417-443.

[B2] Weak convergence of Palais-Smale Sequences for some critical functionals.

[CE] J. Cheeger 83 D. G. Ebin, Comparison Theorems in Riemannian Geometry, North-Holland, Amsterdam, 1975.

[ClmS] R. Coifman, P. L. Lions, Y. Meyer 8 S. Semmes, Compensated compactness and Hardy spaces, J. Math. Pures Appl. 72 (1993), 247-286.

[D] E. DiBenedetto, $C^{1+\alpha}$ local regularity of weak solutions of degenerate elliptic equations, Nonlinear Anal. 7 (1983), 827-850.

[E1] L. C. Evans, Partial regularity for stationary harmonic maps into spheres, Arch. Rat. Mech. Anal. 116 (1991), 101-113.

[E2] - A new proof of the local $C^{1, \alpha}$ regularity for solutions of certain degenerate elliptic P.D.E., J. Differential Equations 45 (1982), 356-373.

[EG] L. C. Evans 63 R. F. Gariepy, Measure Theory and Fine Properties of Functions, CRC Press, Studies in Advances Mathematics, Boca Raton, 1992.

[F] C. Fefferman, Characterizations of bounded mean oscillation, Bull. Amer. Math. Soc. 77 (1971), 585-587.

[FS] C. Fefferman $\mathcal{G}$ E. Stein, $\mathcal{H}^{p}$ spaces of several variables, Acta Math. 129 (1972), 137-193.

[Fu1] M. Fuchs, Some regularity theorems for mappings which are stationary points of the p-energy functional, Analysis 9 (1989), 127-143.

[Fu2] - The blow-up of p-harmonic maps, Manuscripta Math. 81 (1993), 89-94.

[GT] D. Gilbarg 60 N. S. Trudinger, Elliptic Partial Differential Equations of Second Order, Springer-Verlag, New York, 1983.

[HL] R. Hardt $\&$ F. H. Lin, Mappings minimizing the $L^{p}$ norm of the gradient, Comm. Pure Appl. Math. 40 (1987), 556-588.

[HLM] R. HARDt, F. H. Lin $\&$ L. Mou, Strong convergence of p-harmonic mappings (Preprint).

[H1] F. HÉLEIN, Regularity of weakly harmonic maps from a surface into a manifold with symmetries, Manuscripta Math. 70 (1991), 203-218.

[H2] Regularité des applications faiblement harmoniques entre une surface et une variété riemannienne, C. R. Acad. Sci. 312 (1991), 591-596.

[JJ] P. Jones $\mathcal{E}$ J. L. Journé, On the weak convergence in $\mathcal{H}^{1}\left(\mathbf{R}^{d}\right)$, Proc. Amer. Math. Soc. 120 (1994), 137-138.

[L1] S. Luckhaus, Convergence of minimizers for the p-Dirichlet integral, Math. Z. 213 (1993), 449-456.

[L2] - Partial Hölder continuity for minima of certain energies among maps into a Riemannian manifold, Indiana Univ. Math. J. 37 (1988), 349-367.

[M] C. B. Morrey, JR., Multiple Integrals in the Calculus of Variations, SpringerVerlag, New York, 1966.

[MY] L. Mou 6 P. YANG, Regularity for n-harmonic maps, J. Geom. Anal. (to appear).

[P] P. PrICE, A monotonicity formula for Yang-Mills fields, Manuscripta Math. 43 (1983), 131-166.

[R] T. RIVIÉRE, Everywhere discontinuous harmonic maps from $B^{3}$ into $S^{2}$, C. R. Acad. Sci. 314 (1992), 719-723. 
[Sch] R. Schoen, Analytic aspects of the harmonic map problem, Seminar on Nonlinear Partial Differential Equations (S. S.Chern, ed.), MSRI Publications, vol. 2, Springer-Verlag, New York, 1984.

[SU] R. Schoen $\&$ K. Uhlenbeck, A regularity theory for harmonic maps, J. Differential Geom. 17 (1982), 307-335.

[S] S. Semmes, A primer on Hardy spaces, and some remarks on a theorem of Evans and Müller, Comm. Partial Differential Equations 19 (1994), 277-319.

[Sh] J. Shatah, Weak solutions and development of singularities of the $S U(2) \sigma$-model, Comm. Pure Appl. Math 41 (1988), 459-469.

[Ta] H. TAKEuChI, Some conformal properties of p-harmonic maps and a regularity for sphere-valued p-harmonic maps, J. Math. Soc. Japan 46 (1994), 217-234.

[T] P. Tolksdorf, Everywhere regularity of some quasilinear systems with lack of ellipticity, Ann. Mat. Pura Appl. 134 (1983), 241-266.

[U] K.Uhlenbeck, Regularity for a class of non-linear elliptic systems, Acta Math. 138 (1977), 219-240.

\section{TAtiana TORO}

Department of Mathematics

University of Chicago

Chicago, Illinois 60637

E-mail: toro@math.uchicago.edu
Changyou Wang

Department of Mathematics

Rice University

Houston, Texas 77251-1892

E-mail: cywang@math.rice.edu

Received: May 24th, 1994. 